\title{
Creating Sonic Topologies using Electronic Music to develop a new Cosmological Model of Consciousness
}

\author{
Willard G. Van De Bogart 735 11th Ave., Apt.\#1 \\ San Francisco, California 94118 \\ vanflight@gmail.com
}

\begin{abstract}
How electronic music has transformed itself from being a marginal compositional mode of expression for science fiction sound tracks to becoming an integrated sonic art form to enhance emotional and intellectual motivation, as explored by Jóhann Jóhannsson in his sound track for the film, Arrival, is an ongoing process that can lead to a wider view of understanding our place in the universe. Through the use of sonic articulations, the emergent process of scientific exploration in the fields of asteroseismology, nano tunneling microscopes, as well as EM and gravitational fields found in the universe are producing a new sonic tapestry defining the distribution of frequency patterns which form life as we know it. With the sonification and direct recording of cosmic and protein frequencies a new philosophical and cosmological expressions of how consciousness operates in the universe can come about. The spatial domain in which sonic artefacts fill the mind lends insight as to how cognitive spatiality effects sound composition. These new subjective interpretations form a new palette from which an electronic music composer can draw from. By exploring new forms of sonic spatiality a deeper understanding of our cognitive abilities in the universe can come about.
\end{abstract}

Consciousness, synthesizers, topology, composition, space, protein, cosmology, radio telescopes

\section{INTRODUCTION}

Combining scientific studies on the composition and origins of our solar system, our galaxy, the universe and our place in it is constantly being updated as new understanding of how celestial forces interact to create matter and life as we know it. Among those forces which make up our existence are newer fields of study such as gravitational wave astronomy (Billings 2016) and plasma physics (Peratt 2013), or even a hypothetical fifth force named X17 (Lopes 2019), which have emerged as paradigmatic cosmologies which have changed our views from a big bang cosmology to a plasma or electric universe whereby electric, magnetic and gravitational fields hold together the fabric of the universe creating a galactic cybernetic feedback loop. Observing and measuring these forces have raised questions as to what are the mechanisms within the universal quantum field that have been able to create a human species that has gained the ability to be self-reflective and self-reproductive? In contemplating a self-organizing universe, it appears it has the ability to even expand our consciousness in unison with the forces in the universe (Florinsky 2010). Not surprisingly speculation about our origins and our direction as a species has eclipsed anything previously thought possible. We now understand the wave mechanics of energy measured by rates of change, modulation of waves and the dynamic motions seemingly embedded in every aspect of creation. From waves to frequencies to sounds we now know that we exist in a vibratory and highly attenuated oscillation of frequencies on all aspects of interaction with the fundamental forces as currently understood (Geesink and Meijer 2016). One addition to the standard model of the universe is how consciousness is being considered as a fundamental force of nature. A thinking universe has replaced a cold and inert space time continuum. Instead it now appears that a self-organizing field of energy (Jantsch 2019) is responsible for the manifestation of all matter and is embedded on all scales (Storoy 2014). Out of these fields what emerges are photons, solitons, gluons, gravitons and a myriad of forms and a multiplicity of components creating a universe that rings with a beat and pattern that can now be deciphered and transformed into an audible reality mirroring the very structure of the universe. With this in mind it is now possible to embrace an understanding of a participatory universe whereby there is the conception that our own subjectivity and the 
components existing in time and space are somehow inextricably interwoven and connected. Our ability to transpose that unseen and unheard world has now become a new way to experience the formative forces of the universe. From the sonic domain of stars (Handler 2012), to the sounds of planets and subatomic realms both animate and inanimate (Geesink, Meijer 2019), and within the very protein structures of our bodies (Qin, Buehler 2019) is a way to re-create a new tapestry of sound. It is from these sounds we can find expressions for a new cosmology which becomes the palette from which emerges a sentient quantum reality or a state of quantum consciousness providing a quantum aesthetics (Morales 2002) by which to create new forms of art as well as new techniques of scientific investigation.

\subsection{Advent of the synthesizer}

The advent of the electronic music synthesizer enabled the composer for the first time to create with a myriad of frequencies unattainable with traditional instruments. As the frequency structures of the universe on both the micro and macro levels became better understood it became possible to integrate other components of the universe into electronic music compositions such as sonified frequencies from newly discovered celestial bodies as well as advances in the bio and neurosciences. Associating the neuro-structure of the brain with the structural design of the universe has led to even newer fields of study such as acoustic cosmology (Stark 2017), topologies of electrical patterns of the brain and even the sonic structure of consciousness within the universe. Now we have the ability to create new scales based on the very structure of nature (Grady 2017) or algorithms that were designed based on the electromagnetic (EM) frequencies found in animate or inanimate matter (Geesink, Meijer 2019). This research has led this author to produce a new field of study namely neuro-acoustic architecture leading to how we can build cognitive spaces with sound. How sound is interpreted or visualized in the conscious mind is the area

where sonic topologies can be envisioned and created using the electronic synthesizer in real time much like how a piano can create moods and emotions with musical structures similar to the frequencies created with the synthesizer. The sonic architecture created by neuro-acoustics can be further enhanced by applying linguistic fields of thought which support a sound spatiality created by the synthesizer in the further development of neuroacoustic spaces. Phonemic building blocks using frequencies within these sonic topologies gives rise to a new data base of linguistic possibilities whereupon concepts and percepts are created using selected frequencies. Extending these concepts within the neuro-acoustic architecture is accomplished by integrating micro and macro frequency structures that are afforded by the electronic synthesizer, which extends the reach and direction of consciousness created by the electronic synthesizer along with its capabilities to support new sonic topologies or mental soundscapes. Sonic topologies are here referred to as mental spaces created by sound.

\subsubsection{Microtubules and proteins}

The relationship of sound to the building blocks of life in the universe and in turn to the phenomenon of consciousness has been the subject of research by many researchers and scholars. One such researcher is Anirban Bandyopadhyay who has been interested in the interface between the vibrations of the universe and how they directly affect the microtubules in the neurons of the brain. Bandyopadhyay believes that consciousness is being with the universe and that every living system, every conscious being is a part of the music that is played by the universe. The interface of the quantum world directly affecting the molecules of the microtubules led to the discovery of the frequency structure inside the microtubules (Adelaide 2017), (Brown 2017). Further collaboration with Stuart Hameroff and David Penrose led to the Orch Or Theory which suggests that there is a connection between the brain's bio molecular processes and the basic structure of the universe (Hameroff, Penrose 2014). The theory, called "orchestrated objective reduction" suggested that quantum vibrational computations in microtubules were "orchestrated" ("Orch") by synaptic inputs and memory stored in microtubules, and terminated by Penrose which he defined as "objective reduction" ('OR'), hence "Orch OR." The theory further suggests that

consciousness derives from quantum vibrations in microtubules (protein polymers inside brain neurons), which govern neuronal and synaptic functions, and also connect brain processes to selforganizing processes in the fine scale, 'protoconscious' quantum structures of reality (Angelica 2014).

These frequencies found in the microtubules led to further research in the field of protein folding patterns and the frequencies resulting from the folding patterns. The connection of the quantum field interfacing with the brain eventually led to how those frequencies are interpreted in the brain 
leading to a new theory of consciousness which states that the universe and the brain are symbiotically connected. By transposing the $\mathrm{THz}$, $\mathrm{GHz}$ and $\mathrm{MHz}$ frequencies recorded in the nano world of the microtubules into the audible range of humans allowed a new participation with the quantum fields in the universe and those frequencies from our own state of consciousness. For the first time a direct connection was made between thought and vibrations (Raghu 2018). The idea that these sounds were constantly restructuring themselves in our neurons led to conceptualizing a whole new relationship of sound and the way we conceive of it and even how it manifests in our brain.

\section{PROTEINS AND MUSIC}

The next research that dealt with the sounds on a nano scale is the work of Markus Buehler (Figure 1.). from MIT and his work with the sound of proteins. The research team at MIT established a scientific analogy between spider silk and musical composition using Ologs and category theory.

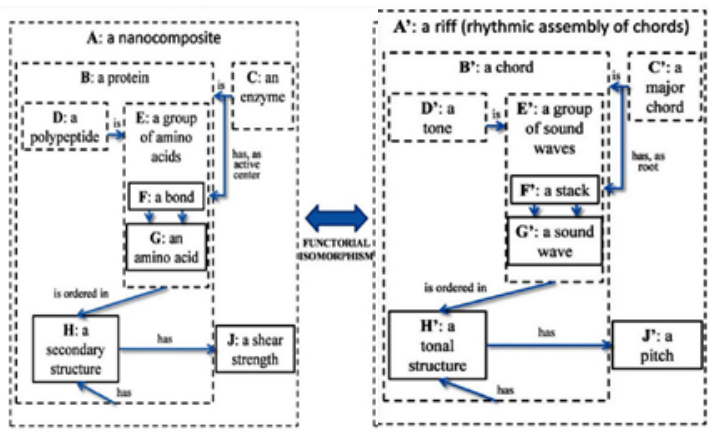

Figure. 1: Functorial analogy of protein materials and musical structure

Buehler's team was analyzing the nano structure of spider silk and making comparisons between the protein structure of the spider strands and musical structures to provide a rigorous mathematical framework for knowledge representation, and the construction of scientific models and data storage. With Ologs and their graphical representation of the organization of proteins and musical structures the team eventually was able to create the sounds of the protein structure of the spider silk. It was from this initial analysis of the spider silk that a concerted effort was made to determine the sounds made by over 100,000 thousand proteins leading to the development of the protein synthesizer (Giesa et.al., 2011).

\subsubsection{Protein Synthesizer}

After computing the spider silk protein into sound, a computational algorithm was designed to extract the natural frequencies of all proteins in the protein data bank (PDB), which is a database for 3D structural data of large biological molecules, such as proteins and nucleic acids, and then proceed to the description of sonifying them and implementing them in a playable instrument Figure 2. (Qin and Buehler 2019).

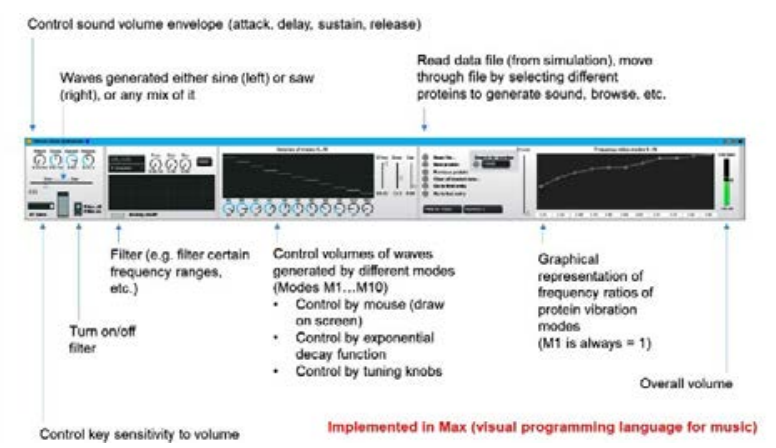

Figure 2: Detail of the Protein Synthesizer control panel.

For the first time it was possible to integrate the sounds of proteins directly into electronic synthesizer compositions. Given the fact that external electromagnetic frequencies affect the vibration of the amino acids that are responsible in creating proteins (Meijer and Geesink 2017) it was now possible to create sounds that were a part of the protein structure of the human organism. What was achieved was to automatically compute the vibrational spectra of more than 100,000 proteins available in the Protein Data Bank. Then by using the resulting frequency spectra of the proteins they were able to generate audible sound by overlaying the molecular vibrations and translating them to the audible frequency range using the music theoretic concept of transpositional equivalence. The method, implemented as a Max audio device for use in a digital audio workstation (DAW), provided vibrational signatures of the protein structures. This combination of EM fields affecting protein molecules as well as the sounds made by the proteins gave rise to realizing that the vibrational forces in the universe were responsible for the vibration patterns holding together the proteins in the human organism. The interplay of such discrete electromagnetic radiation frequencies in the guiding of cellular function, also makes clear that life systems can, in principle, obtain sufficient external information to further explain their integral life complexity. 


\section{SONIC AND CONCEPTUAL TOPOLOGIES}

The combination of frequency patterns created by electronic synthesizers and a new protein synthesizer developed by Qin and Buehler (2019) can influence and create conceptual patterns within the mind's eye. This approach to creating electronic music with mixed frequency patterns is a technique by which a composer can interact with these conceptual patterns when developing electronic music soundscapes. These sonic and conceptual patterns arise within a neural topological space or a state of consciousness revealing how sound and even language construction can create not only newer visual representations within the mind's eye but also the creation of sounds which are directly created from using these new conceptualizations as source material when creating electronic music. Sound naturally creates a spatial dimension within the brain known as binaural hearing (Hölzl 2003) as well as an inner relationship to sound such as an inter-aural phenomenon whereby the brain tries to perceive where the sounds originate from so as to help locate oneself in the physical world. However, with conceptual topologies aided by neuro-acoustic stimulation shapes and ideas can appear in the conceptual field to help reference the source of sound outside the body as well as creating an inner neuro-spatiality or inner acoustic architecture to create newer cognitive realizations. Karlheinz Stockhausen was a pioneer in the topology of space for his sounds and felt it was necessary to make sound move in any direction with any given speed and in any given space (Cott 1973 p. 203). The recent utilization of protein sounds is a new addition to the sonic palette. By sonifying the very wave dynamics of protein molecules, a never before neuro-acoustic stimulus is being experienced not from a manmade instrument but integrating the natural sounds which are part of our own bodily functions. These newly discovered resonant frequency distribution patterns coming from the proteins are then experienced by the brain in a symbiotic relationship between cognitive activity as well as experiencing a resonant relationship with the sonified protein. This new brain dynamic concept has been explored at the Qualia Research Institute (Johnson 2009) where effort is being made to discover the interconnections in the entire brain to bring forth a new ontology for brain dynamics combining neural resonance and neural acoustics.

\subsection{Language and conceptual topologies}

Conceptual topologies and models of consciousness are closely linked. The phenomenality or the quality of a conscious state has been explored as a way to give a framework by which to understand the space in which our conscious thoughts take place (Prentner 2014). Prentner developed six central concepts, Figure 3., in a hexagram to show the relationships that language could have and how the interrelationship of the extension of the meaning of the key words in the hexagram continue to form a complex and continuous relationship of ideas.

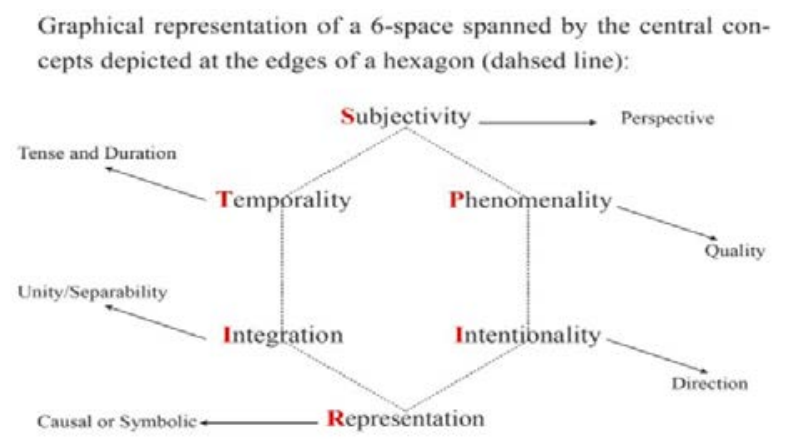

Figure 3: Model of language in a 6-space hexagram from "Toward a Science of Consciousness" Tucson, AZ 2014

This is an example of another conceptual topology that when combined with a sonic topology of aural spatiality creates an internal subjective field where language and sound spaces intermingle. This combination of language usage or idea creation infused in an aural topology is the area the composer can utilize when composing electronic music. Creating new sounds that are exploring topological space is a way for the mind to explore the hidden dimensions that lurk within ourselves (Hoffmann-Dietrich 2013). The integration of the spaces that occur when proteins begin to fold has been an area of exploration that has led HoffmannDietrich to explore the many hidden spaces the proteins create. As was pointed out by Buehler from MIT each folded protein creates its own sound. By introducing the protein sound into sonic topological spaces as well as how language may be modeled in a subjective space introduces many new parameters that can be explored from a compositional framework. How each protein sound conveys its own frequency structure is a representation of the space that the protein is creating coupled with the frequency distribution which creates the completion of the folded protein. What takes place with the creation of live electronic music is a new representation of the spatiality that is experienced from the internal visual patterns which 
are also created in real time by creating conceptual topologies which can appear in the mind's eye as a result of weaving the multiple spatial domains of sound and language.

\section{COSMOLOGICAL MODELS OF CONSCIOUSNESS}

The next area that unfolds or emerges from these spatial topologies created by sound and concepts based on language are interpretations of the very space of the universe we exist in. Living as we do in a vast expanse of space in the universe, which has had a direct impact on how our bodies and minds have been created (Florinsky), is how we are able to comprehend the relationship between sound and language. Today new spatial settings are influenced not only by the sounds inside our bodies, such as the nano world of the molecules making up our protein structure, but also in the realm of how our thoughts are created in the neuro-acoustic spaces where sounds and language are derived from. Once we begin to create music and ideas, which is reflective of the nano world as well as how that world effects the larger macro existence that we derive our thinking from, is then that it is only natural

that we have the opportunity to begin to experience newer aural spaces within our own mental framework as well as exchanging that framework with the listener. One such model that includes sound with cosmology is acoustic cosmology where the very definition of our existence can be experienced from the different sound structures which make up the universe (Starks 2017). Starks' mission is to show that there is a musical equivalent to the curvature of space-time in the universe. The recognition of the fact that we use newer created digital sounds from the micro structures of our bodies as well as those found within astronomical objects leads to a new theory proposed by Stark which he calls a "soniverse" whereby an explanation for existence can be interpreted from how we arrange sound using a micro-structured approach. Explaining existence from a cosmological perspective has been attempted by countless authors. How the biological brain constructs subjective consciousness is a question many scholars have attempted to answer (King 2011). How sound is incorporated into this quest has been the subject of this paper. To suggest there would be a deeper understanding of how we use our consciousness as a result of conceptual topologies created by sound and language constructs has already been briefly discussed. Within this brief discussion it is evident that this pursuit to understand our place in the universe has been a quest whereby sound has played a primary role in this description of sonic topologies for describing a working cosmology. The use of models of consciousness has also played an important role in understanding how sound can be used in a new cosmological framework. The key aspect in this pursuit of a new cosmological model of the universe is the realization that it must filter through our own comprehension of reality. In as much as the electronic music composer is represented in this paper sound has been the focus whether it be created and experienced by the composer or it is the listener who as a conscious participant experiences similar thoughts that would lead to creating a newer model of one's place the universe. The torus model shown in Figure 4. Is a way to show how a torus can be

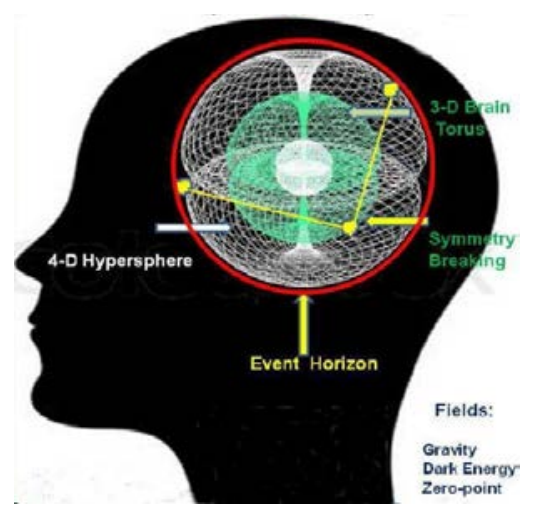

Figure.4: Field receptive, event horizon model of consciousness (Meijer, Geesink 2017).

visualized within the event field of the universe and is an example of how the flow of energy moves within an electromagnetic universe. The integration of scientific principles and artistic utilization is a way by which current understanding can be integrated across many disciplines.

\section{AESTHETIC DIMENSIONS OF CONTEMPORARY ELECTRONIC MUSIC}

Applying an aesthetic to electronic music which has now evolved on so many fronts with capabilities to alter sounds with new musical scales as well as sounds never before heard opens up a vast interpretive discussion on whether music will remain associated with conventional music theory or will embark on a newer expression for its definition. This paper has touched upon new inner aural spatiality's as a source for composing new sounds that could ultimately lead to exploring the depth of space as interpreted with sonic landscapes and the concomitant sensibilities experienced from such sounds. With the advent of a new instrument such 
as the protein synthesizer developed at MIT what we can experience now are new tonal structures made by the sonification of the wave dynamics within the molecular structure of the proteins which constitutes the very building blocks which gives us life. Mixing and rearranging these sounds and creatively selecting sounds made from electronic synthesis is a totally new form of sonic expression which has little in common with traditional music. What does exist, however, is an aurality of constructing a conceptual; sonic space predicated on sonic elements that come from the universe we live in as well as how those sounds from space have been responsible for creating our mental space that allows us our own functionality. Movement of sound is another component of the electronic music sphere. Once a synthesizer is turned on it only takes a short time before a sound will be heard. It can be a specifically designed sound from a vast selection of modular components, or it can be a readymade sound just by giving amplitude to an oscillator. But unlike traditional instruments where a bow is pulled back and forth or fingers are sliding on strings or air is being pushed through various wind instruments the synthesizer is an instrument where frequencies of many types are possible instantly. Those frequencies have been discussed and act as guides for their use in this paper by associating the frequencies with conceptual topological spaces created by incorporating a subjective component that reaches into another world of quantum exchanges with sounds from realms we never before have experienced. The movement of those sounds as they move within the universe and our body and mind are constant and never stop. The electronic composer has the capability to play along with the sounds of the universe in a specific way which is the aesthetic choice once the acceptance of the electronic sounds represent more than a frequency but frequencies that are patterned after the movement of the natural forces in the universe. The aesthetics of electroacoustic music has been less interpreted from a subjective point of view and more from an objective point of view (Fronzi 2018 p. 1.) Electronic music, therefore, has to be internalized as a means to make newer associations with the quest of seeking who we are in this vast universe. Our electronic sound instruments are pushing us further and further into realms of the universe never before thought possible and signals are being recorded and sent back to our databases for further inspection and decipherment. China's FAST radio telescope Figure 5. was specifically designed to see if intelligent life was making sounds in the universe (Gibney, 2019).

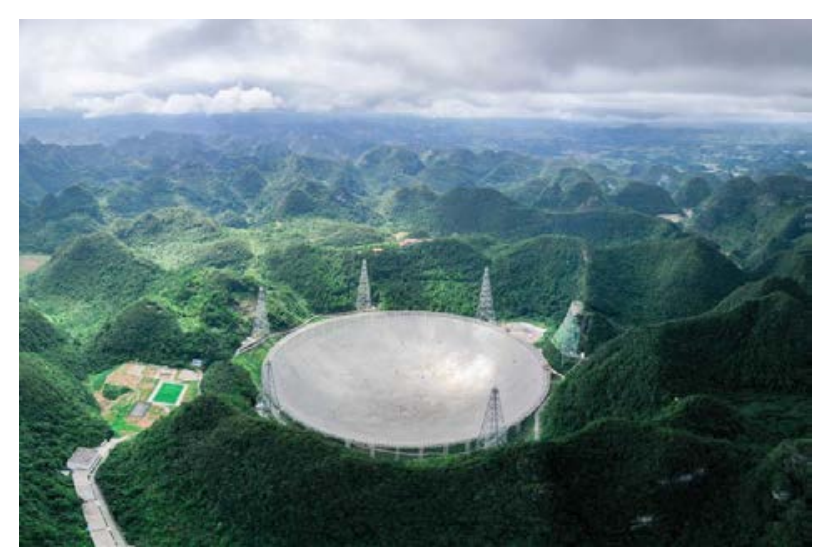

Figure. 5: The Five-hundred-meter Aperture Spherical Radio Telescope (FAST)

The FAST radio telescope is an example of listening to sounds from the depths of space much like the new electronic music composer in the first quarter of the 21st century has begun to peer into the depths of the human body to determine how the frequencies from space affect the very development of our body. These new electronic music composers are creating new topologies made from the building blocks of language such as concepts and phonemic samples from languages around the world. The ideal art and technology interface is having the new electronic music composer listen to the sounds from outer space with a radio telescope and compare the aural dimensions experienced when composing with new sonic topologies which include sounds from space. This is where new frontiers will open up when we use the creative mind sourcing sounds from the micro and macro dimensions and compare them with how our new instruments that are exploring space are integrated with the sounds the composer is making on earth.

\section{CONCLUSION}

Combining the three areas of topology, sound and cosmology were chosen as a demonstration on how the electronic music composer is entering into the scientific realms where research in the space sciences as well as the biological and neuro sciences are revealing a vibratory physics that crosses all disciplines. Entering into these new sciences will bring newer discoveries that can enhance the way synthesizers are designed and adapted to interfacing with the new sciences. This art and technology interface is growing exponentially opening up entirely new areas for sonic exploration which will in all likelihood bring us closer to the very design elements which constitute how our own bodies are assembled. Sound art will evolve to the point that sonic realizations 
representing our body will be a normal practice much like 3D printing has evolved. Quantum computers will reveal new patterns that can be incorporated in electronic synthesizers where making sonic human bodies will be an everyday sonic practice or even traveling will be made possible by resonating with the plasma fields in space to transport ourselves using newly designed signal transmission telescopes. It is an open future that affords experimentation on a wide scale of activities which has the potential to alleviate the stress we have placed on the environment and social structures. The key is experimenting with new integrative ideas to uncover the complexities of a highly integrated eco/space system. It is time artists and scientists emerge as the harbingers of innovation to foster a sustainable planet so we can venture out into space with our species knowing that we solved how to integrate life generating fields to sustain our existence. It sounds like an ambitious projection but the alternative of keeping the status quo or doing nothing will not advance our ideas into the future.

\section{REFERENCES}

Angelica. A. (2014) Discovery of quantum vibrations in microtubules inside brain neurons corroborates controversial 20-year-old theory of consciousness, Kurzweil Newsletter. Available from https://www.kurzweilai.net/discovery-of-quantumvibrations-in-microtubules-inside-brain-neuronscorroborates-controversial-20-year-old-theory-ofconsciousness (09 September 2019).

Adelaide, V. (2017) Beautiful Humans. Available from: https://www. beautifulhumans.info/anirbanbandyopadhyay/ (09 September 2019).

Billings, L. (2016) The Future of Gravitational Wave Astronomy, Scientific American Available from: https://www.scientificamerican.com/article/thefuture-of-gravitational-wave-astronomy/ (12 February 2016).

Brown, W. (2017) Confirmation of Quantum Resonance in Brain Microtubules, Available from: https://resonancescience.org/confirmation-ofquantum-resonance-in-brain-microtubules/ (09 September 2017).

Chandler, D.L. (2019) Translating proteins into music, and back MIT News/MIT News Office.Available from http://news.mit.edu/2019/translating-proteins-music0626 (28 September 2019).
Cott, J. (1973) Stockhausen: Conversations with the composer. New York: Simon and Shuster.

Florinsky, V.I., (2010) Man and the Geosphere. Nova Science Publ Avalilabishers, Inc. Available from: http://iflorinsky.impb.ru/Florinsky-2010d.pdf (5 December 2019).

Fronzi, G. (2018) Aesthetics of Electroacoustic Music, "International Lexicon of Aesthetics", Spring 2018 edition

https://lexicon.mimesisjournals.com/archive/2018/sp ring/AestheticsOfElectroacousticMusic.pdf (29 September 2019).

Geesink, H.J.H., Meijer, D.K.F. (2016) Quantum Wave Information of Life Revealed: An Algorithm for Electromagnetic Frequencies that Create Stability of Biological Order, with Implications for Brain Function and Consciousness. NeuroQuantology, Volume 14 (Issue 1/March). pp 106-125.

Geesink, H.J.H., Meijer, D.K.F. (2019) A novel biophysical quantum algorithm predicts superconductive properties in animate and inanimate systems. Researchgate.net. Available from: https://www.researchgate.net/publication/33190297 7 A novel biophysical quantum algorithm predict s superconductive properties in animate and inanimate systems (27 September 2019).

Gibney, E. (2019) Gigantic Chinese telescope opens to astronomers worldwide Nature Available from: https://www.nature.com/articles/d41586-01902790-3 (29 September 2019)

Giesa, T., Spivak, D.I., Buehler, M.J. (2011) Reoccurring Patterns in Hierarchical Protein Materials and Music: The Power of Analogies, BioNanoScience. Volume. 1. Issue 4/December, pp 153-161.

Grady, K.. (2019) The Wilson Archives Available from: http://www.anaphoria.com/wilson.html (29 September 2019).

Handler, G. (2012) Asteroseismology, Copernicus Astronomical Center/arXiv.org Available from: https://arxiv.org/pdf/1205.6407.pdf (23 September 2019).

Hoffmann-Dietrich (2013) Hidden Topology of Life: Life and Space. Available from: http://www.johoffmann.com/J.HD. Hidden\%20Topol ogy\%20of\%20Life.pdf (29 September 2019). 
Jantsch, E. (2019) Cosmic Evolution: The SelfOrganizing Universe. Available from:

http://palaeos.com/cosmic evolution/selforg.html

(29 September 2019).

Johnson, M. (2009) Toward a new ontology of brain dynamics: neural resonance + neuroacoustics Qualia Research Institute. Available from:

https://opentheory.net/2009/11/toward-a-newontology-of-brain-dynamics-neural-resonanceneuroacoustics/ (28 September 2019).

King, C. (2011) Cosmological Foundations of Consciousness. Available from:

https://www.dhushara.com/cosfcos/cosfcos2.html (5 December 2019)

Lopes, A. (2019) Fifth Force of Nature: The Plot Thickens for a Hypothetical "X17"

Particle.SciTechDaily Available from:

https://scitechdaily.com/fifth-force-of-nature-the-plotthickens-for-a-hypothetical-x17-particle/ (5 December 2019).

Meijer, D.K.F., Geesink, H.J.H. (2017)

Consciousness in the Universe is Scale Invariant and Implies an Event Horizon of the Human Brain. NeuroQuantology, Vol. 15, No. 3/September. pp 4179. Available from:

https://www.neuroquantology.com/index.php/journ al/article/view/1079 (23 September 2019).

Morales, G. (2002) "Overcoming the Limit Syndrome". In: Caro, M.J., Murphy, J.W. The World of Quantum Culture. Westport, Connecticut: Praeger. pp.1-34.
Peratt, A.L. (2013) Plasma Universe The IEEE Nuclear and Plasma Sciences Society. Available from: https://plasmauniverse.info/ (29 September 2019).

Prentner, R. (2014) Conceptual Topologies and Models of Consciousness Academic.edu. Available from:

https://www.academia.edu/19286440/Conceptual T opologies and Models of Consciousness (28 September 2019).

Raghu, M. (2018) A Study to Explore the Effects of Sound Vibrations on Consciousness. Available from:

http://www.hrpub.org/download/20180730/IJRH219290514.pdf (5 December 2019).

Stark, G. (2017) Adventures in Acoustic Cosmology. Royal Astronomical Society Available from: http://www.binarydust.org/author/admin/ (29 September 2019).

Storoy, D. (2014) David Bohm, Implicate Order and Holomovement. Science and NonDuality. Available from:

https://www.scienceandnonduality.com/article/davi d-bohm-implicate-order-and-holomovement (27 September 2019).

Qin, Z., Buehler, M.J. (2019) Analysis of the vibrational and sound spectrum of over 100,000 protein structures and application in sonification. ScienceDirect Available from:

https://www.sciencedirect.com/science/article/Pii/S2 352431618302414 (18 September 2019). 